\title{
PULSATION MODES AND PERIOD-LUMINOSITY-COLOR RELATIONS FOR THE SHORT-PERIOD $\delta$ SCUTI STARS
}

\author{
MICHEL BREGER \\ University of Texas at Austin and McDonald Observatory
}

\begin{abstract}
Due to recent contradictory results, the period-luminosity-color relation of $\delta$ Scuti stars has been examined by a maximum likelihood method. $\delta$ Scuti stars are found to definitely obey a period-luminosity-color relation. The derived coefficients are very similar to those derived for RR Lyrae variables. Care has to be taken to allow for the large mass differences between the population I variables, such as $\delta$ Scuti stars, and population II ultrashort period pulsators.

The value of the pulsation constant $Q$ is derived for a large sample of $\delta$ Scuti stars by calibrating the narrowband $u v b y$ indices in $T_{\text {eff }}$ and $\log g$ from a complete set of model atmospheres (ATLAS). Most variables pulsate in the fundamental mode or the first overtone. Analogous to RR Lyrae stars, the division into different pulsation modes may be determined by color. For the main-sequence pulsators, this is not in agreement with theoretical transition period results which predict that no $\delta$ Scuti stars should pulsate in the fundamental (radial) mode. Should non-radial modes be important for $\delta$ Scuti stars, then the non-radial pulsation modes need to have $Q$ values quite similar to radial modes.
\end{abstract}

\section{DISCUSSION}

Irwin: How were the original $\mathbf{M}_{\mathbf{v}}$ 's determined for these $\delta$ Scuti stars?

Breger: For cluster members, and there are quite a few of those, they were derived from cluster membership. For a few stars they were derived by a new calibration of $u b v y \beta$ photometry, which Crawford has made and which we have made.

Irwin: You said later that you used trigonometric parallaxes somewhere along the line.

Breger: Yes. For the dwarf Cepheids, because a photometric calibration assumes that you have a normal mass for the luminosity (I don't think you have a normal mass), photometric calibrations break down.

Rodgers: What are the effects of differential metallicity on the calibration, are they large?

Breger: You mean on the observed period?

Rodgers: No, on the calibration of effective gravity as a function of $c_{1}$.

Breger: I have not investigated this; I think Dr Bell can say something on that. I've used normal solar abundance models. The effects are likely not to be large, though. An Am star may be an extreme abundance star, in fact an Am star seems to fit these calibrations. 